\title{
EFEITO DE CARGAS APLICADAS E PROFUNDIDADES DE SEMEADURA NO DESENVOLVIMENTO DA CULTURA DO FEIJÃO EM SISTEMA PLANTIO DIRETO
}

\author{
Effect of loads applied and depths of sowing in the \\ development of bean culture in no-tillage system
}

\author{
Alcir José Modolo, Emerson Trogello², Anderson Luis Nunes ${ }^{3}$, Haroldo Carlos Fernandes ${ }^{4}$, \\ João Cleber Modernel da Silveira ${ }^{5}$, Marcos Paulo Dambrós ${ }^{2}$
}

\begin{abstract}
RESUMO
Dentre os fatores primordiais para a germinação estão a umidade, a temperatura e a aeração do solo. É importante citar que esses fatores são diretamente influenciados pelo estado de compactação do solo ao redor da semente. Objetivou-se, neste trabalho, estudar os efeitos da combinação entre profundidades de semeadura e cargas aplicadas pela roda compactadora da semeadoraadubadora sobre o desenvolvimento da cultura do feijão, em sistema plantio direto. Foi utilizado o esquema fatorial no delineamento em blocos ao acaso, composto por doze tratamentos (três profundidades de semeadura e quatro níveis de cargas aplicadas pela roda compactadora) e quatro repetições. Os resultados mostraram que a profundidade de semeadura influenciou, significativamente, o índice de velocidade de emergência, os estandes inicial e final de plantas e o rendimento da cultura. As diferentes cargas aplicadas pela roda compactadora não influenciaram significativamente as variáveis avaliadas.
\end{abstract}

Termos para indexação: Mecanização agrícola, semeadura, emergência.

\begin{abstract}
Among the primordial factors for the germination are humidity, temperature, and aeration of the soil. It is important to mention that these factors are directly influenced by the soil compaction state around the seed. The present paper had the aim of studying the effects of the combination between depths of sowing and loads applied by press wheel of the seeder upon the development of bean culture, in a no-tillage system. Factor schedule was applied to the delineation in random blocks composed by twelve treatments (three depths of sowing and four levels of loads applied by the press wheel) and four replications. The results showed that depth of sowing significantly influenced the index of emergency speed, the initial and final stands of plants and yield of culture. The different loads applied for the press wheel did not significantly influence the evaluated variables.
\end{abstract}

Index terms: Agricultural mechanization, sowing, emergency.

(Recebido em 14 de janeiro de 2009 e aprovado em 14 de julho de 2009)

\section{INTRODUÇÃO}

Apesar da importância da cultura do feijão (Phaseolus vulgaris L.), a produtividade média nacional é de aproximadamente $500 \mathrm{~kg} \mathrm{ha}^{-1}$, enquanto, a de outros países como Estados Unidos, Turquia e Japão é 1.486, $1.471 \mathrm{e} 1.420 \mathrm{~kg} \mathrm{ha}^{-1}$, respectivamente. Atualmente, o Brasil dispõe de cultivares com potenciais de rendimento de até $3.600 \mathrm{~kg} \mathrm{ha}^{-1}$ (Rezende et al., 2004). Entretanto, em razão de uma série de fatores relacionados à semeadura, à germinação, à planta, ao ambiente e às práticas de manejo, ainda depara-se com níveis de produtividade abaixo da média nacional que se encontra ao redor de $500 \mathrm{~kg} \mathrm{ha}^{-1}$.

Nas culturas anuais a operação de semeadura se reveste de grande importância, pois possíveis problemas durante essa etapa só poderão ser detectados após a emergência, conduzindo a perdas significativas de produção.

As rodas compactadoras são dispositivos que melhoram o contato solo-semente por meio da aplicação de pressão exercida lateralmente e sobre a linha de semeadura, provocando leve compactação e deixando que o solo, diretamente sobre as sementes, apresente friabilidade suficiente de forma a envolvê-las completamente, sem, no entanto, permitir a formação de crostas, favorecendo emergência das plântulas.

De acordo com Silva et al. (2008), um dos fatores a serem considerados em uma operação de semeadura é a profundidade de deposição das sementes, que pode afetar sua germinação, sendo condicionada pela temperatura, teor

1Universidade Tecnológica Federal do Paraná/UTFPR - Via do Conhecimento km 01 - Cx. P. 571 - 85501-970 - Pato Branco, PR - alcir@utfpr.edu.br

2 Universidade Tecnológica Federal do Paraná/UTFPR - Pato Branco, PR

${ }^{3}$ Universidade Federal do Rio Grande do Sul/UFRGS - Porto Alegre, RS

${ }^{4}$ Universidade Federal de Viçosa/UFV - Campus Universitário - Viçosa, MG

${ }^{5}$ Centro Federal de Educação Tecnológica de Goiás/CEFET - Rio Verde, GO 
de água e tipo de solo, dentre outros fatores. A semente deve ser depositada a uma profundidade que permita um adequado contato com o solo úmido, resultando em elevado percentual de emergência.

Brown et al. (1996) e Silva (2002) relataram que no processo de semeadura de culturas anuais, o condicionamento físico do solo ao redor das sementes reveste-se de grande importância para o bom desenvolvimento inicial da cultura, assegurando uma população adequada de plantas. Phillips \& Kirham (1962) afirmaram que um condicionamento inadequado nessa fase, pode limitar o desenvolvimento das plantas em estádios posteriores de crescimento. Um dos fatores da baixa produtividade está na dificuldade de se estabelecer a população ideal de plantas por ocasião da semeadura, em razão dos vários fatores que impedem a germinação normal de parte das sementes. Dentre os fatores que condicionam o meio ambiente do solo, a umidade, a temperatura e a aeração são primordiais para a germinação (Nabi et al., 2000).

Vários autores têm procurado estudar a relação máquina-solo-planta em ensaios de semeadura, utilizando rodas compactadoras. Furlani et al. (2001) combinando três profundidades de semeadura da cultura do milho com quatro níveis de compactação do solo sobre as sementes, não encontraram influência desses fatores sobre o número médio de dias para emergência das plântulas.

Modolo et al. (2008), avaliando os efeitos da combinação entre teores de água do solo e cargas aplicadas pela roda compactadora da semeadora-adubadora sobre o contato solo-semente, em sistema plantio direto na cultura da soja, concluiram que as cargas aplicadas pela roda compactadora elevaram a densidade do solo e a resistência à penetração no plano vertical da linha de semeadura abaixo da profundidade de semeadura, quando comparado com os valores obtidos antes do plantio. Os autores observaram ainda que a velocidade de emergência das plântulas de soja foi afetada pelas cargas aplicadas e pelos teores de água do solo e que a carga máxima de $140 \mathrm{~N}$, aplicada pela roda compactadora sobre a semente, causou encrostamento superficial do solo, retardando a emergência das plântulas.

Cortez et al. (2005) citam que o uso de cargas verticais de $200 \mathrm{~N}$ sobre as rodas compactadoras diminuiu a velocidade e a porcentagem de emergência para a cultura do algodoeiro, e que cargas verticais em torno de 100 a 150 $\mathrm{N}$ trazem benefício ao desenvolvimento inicial das plântulas.

Silva et al. (1991) verificaram a influência de quatro diferentes modelos de rodas compactadoras sobre o condicionamento físico do solo, utilizando-se três níveis de compactação condizentes com o processo de semeadura, e afirmaram que as mesmas alteram o comportamento hídrico do solo na região de semeadura, além de elevar a densidade e a resistência à penetração no plano vertical da linha de semeadura. A velocidade de emergência e emergência total das plantas de feijoeiro foram afetadas pelas pressões de compactação. À medida que a compactação aumentou, as plântulas tiveram sua emergência retardada e diminuída para todas as rodas testadas.

Dentro do contexto apresentado, neste trabalho objetivou-se estudar os efeitos da combinação entre profundidades de semeadura e cargas aplicadas pela roda compactadora da semeadora-adubadora sobre o desenvolvimento e rendimento da cultura do feijão, em sistema plantio direto.

\section{MATERIAL E MÉTODOS}

O experimento foi desenvolvido na Área Experimental da Universidade Tecnológica Federal do Paraná, em Pato Branco - PR, em um solo classificado pela Embrapa (1999), como sendo Latossolo Vermelho Aluminoférrico com relevo ondulado e textura argilosa, sendo o clima subtropical. A área vem sendo manejada sob sistema plantio direto a mais de 10 anos, com a rotação de culturas, soja ou milho como culturas de verão e aveia mais azevém em cobertura de inverno. O teor médio de água e de massa específica no solo na profundidade de 0 - 0,10 m, foram de $0,30 \mathrm{~kg} \mathrm{~kg}^{-1}$ e $1,25 \mathrm{Mg} \mathrm{m}^{-3}$, respectivamente.

Utilizou-se o esquema fatorial, no delineamento em blocos ao acaso composto por doze tratamentos e quatro repetições. A área foi dividida em quatro blocos casualizados, totalizando quarenta e oito parcelas experimentais, cada uma com área de $120 \mathrm{~m}^{2}$ (4,0 x 30 m). Os doze tratamentos foram compostos pela combinação entre três profundidades de semeadura (P1, P2 e P3), correspondentes a 3,0; 5,0 e 7,0 cm, respectivamente, e quatro níveis de cargas aplicadas pela roda compactadora (C1, C2, C3 e C4), correspondentes a 0, 60, 110 e 190 N, respectivamente.

Utilizou-se a cultivar de feijão UTF-6, produzida pela UTFPR, com ciclo médio de 90 dias em uma densidade de semeadura de 17 sementes por metro linear e espaçamento entre linhas de 0,45 m. A adubação no momento da semeadura constituiu-se de $300 \mathrm{~kg} \mathrm{ha}^{-1}$ da formulação 08-20-20. O controle de insetos e pragas foi realizado aos 18 e aos 33 dias após a semeadura com a utilização do inseticida Endossulfan, na dose de 1,0 $\mathrm{L} \mathrm{ha}^{-1}$ e com volume de 
aplicação de $200 \mathrm{~L} \mathrm{ha}^{-1}$ de calda. As plantas daninhas foram controladas com herbicida Robust aos 20 dias após a emergência do feijão, utilizando a dose de 1,0 $\mathrm{L} \mathrm{ha}^{-1}$.

Para a semeadura do feijão, utilizou-se um trator New Holland, modelo TL75E, 4x2 TDA, com potência máxima de $57,4 \mathrm{~kW}(78 \mathrm{cv})$ no motor a $2.400 \mathrm{rpm}$ e uma semeadora-adubadora de precisão marca Vence Tudo, modelo SA 14600 de arrasto, com mecanismos sulcadores do tipo haste, para fertilizantes e discos duplos defasados, para sementes, e com mecanismos de cobertura e compactação do tipo roda de borracha, convexa, com 330 $\mathrm{mm}$ de diâmetro e $170 \mathrm{~mm}$ de largura.

Os quatro níveis de cargas aplicadas pela roda compactadora sobre a semente foram obtidos por meio da adição de massas metálicas sobre a roda compactadora. $\mathrm{O}$ pino que fixa a roda compactadora foi retirado de modo que esta ficasse livre, e toda a carga fosse aplicada diretamente sobre a roda (Figura 1).

O índice de velocidade de emergência de plântulas (IVE) foi avaliado em um comprimento de 10 metros na linha central de semeadura. O número de sementes inicial foi determinado de acordo com a uniformidade de distribuição da semeadora-adubadora. A contagem das plântulas foi realizada diariamente até que o número de plântulas emergidas se apresentasse constante. Cada planta foi considerada emergida, a partir do instante em que a mesma rompeu o solo e pôde ser vista a olho nu de algum ângulo qualquer. A partir dessas contagens, expressou-se o IVE, utilizando-se a equação (1), adaptada de (Maguire, 1962).

$$
\operatorname{IVE}=\frac{E_{1}}{N_{1}}+\frac{E_{2}}{N_{2}}+\ldots \frac{E_{n}}{N_{n}}
$$

Em que:

IVE = índice de velocidade de emergência;

$\mathrm{E}_{1}, \mathrm{E}_{2}, \mathrm{E}_{\mathrm{n}}=$ número de plântulas emergidas, na primeira, segunda, ..., última contagem;

$\mathrm{N}_{1}, \mathrm{~N}_{2}, \mathrm{~N}_{\mathrm{n}}=$ número de dias após a semeadura à primeira, segunda, .... última contagem.

O estande inicial de plantas foi avaliado a partir do momento que se obteve um número constante de plantas emergidas e a contagem do estande final de plantas foi realizado antes da colheita. A avaliação do número de legumes por planta foi realizada no momento da colheita, sendo coletados os legumes de 10 plantas da linha central de semeadura. Dessas plantas foram coletados 30 legumes aleatórios para realizar a contagem do número de grãos por legume.

$\mathrm{O}$ rendimento médio $\left(\mathrm{kg} \mathrm{ha}^{-1}\right)$ foi obtido pela pesagem dos grãos colhidos na linha de avaliação e posterior extrapolação para um hectare, sendo a colheita realizada manualmente.

Os resultados obtidos foram tabulados e submetidos à análise de variância, conforme os níveis profundidade de semeadura e de cargas aplicadas pela roda compactadora, utilizando-se o programa computacional ASSISTAT. Quando o valor do teste F mostrou-se significativo a $5 \%$ de probabilidade de erro, aplicou-se o teste Tukey para comparação das médias.

\section{RESULTADOS E DISCUSSÃO}

\section{Índice de velocidade de emergência, estandes inicial e final de plantas}

Com relação ao índice de velocidade de emergência das plântulas (IVE), pôde-se observar que, dentre todos os fatores e interações estudados, apenas a profundidade de semeadura afetou de forma significativa a emergência das plântulas de feijão (Tabela 1). A profundidade P2 $(5,0 \mathrm{~cm})$ resultou em maior IVE quando comparado com as profundidades P1 $(3,0)$ e P3 $(7,0 \mathrm{~cm})$.

Os menores valores de IVE encontrados para as profundidades P1 e P3 podem estar associados a deficiências do fechamento do sulco ocorrido durante a semeadura na profundidade $\mathrm{P} 1$, permitindo que a semente ficasse descoberta, ocasionando, dessa forma, um contato inadequado entre o solo e a semente, dificultando a absorção de água pela semente, retardando, assim, o processo de emergência e reduzindo a percentagem de emergência das plântulas. Enquanto que, na profundidade $\mathrm{P} 3$, deve-se, possivelmente, a maior espessura da camada de solo pelo qual a plântula deve romper para emergir.

Dellicer (1984), em seu experimento com a cultura do feijoeiro nas profundidades $(2,0 ; 5,0 ; 7,0$ e 10,0 cm) e cargas verticais $(0,150,300 \mathrm{~N})$, observou que em relação à emergência a profundidade foi significativa para as camadas de 2,0 e 5,0 cm, em relação a camada de 10,0 cm. Para as cargas observou que apenas a de $300 \mathrm{~N}$ diferiu das demais em relação à emergência. Grotta et al. (2007) verificaram que a emergência de plântulas de soja não foi influenciada pela profundidade de semeadura e pela carga vertical aplicada sobre a fileira de semeadura.

Para os estandes inicial e final de plantas (Tabela 1), pode-se observar que de todos os fatores e interações estudados, apenas a profundidade de semeadura apresentou diferenças significativas, sendo que as profundidades P2 e P3 resultaram em maior estande inicial e final de plantas, enquanto que, a profundidade P1 indicou o menor estande, evidenciando um menor contato entre solo-semente, ocasionando maior exposição da semente as condições inadequadas a germinação. 


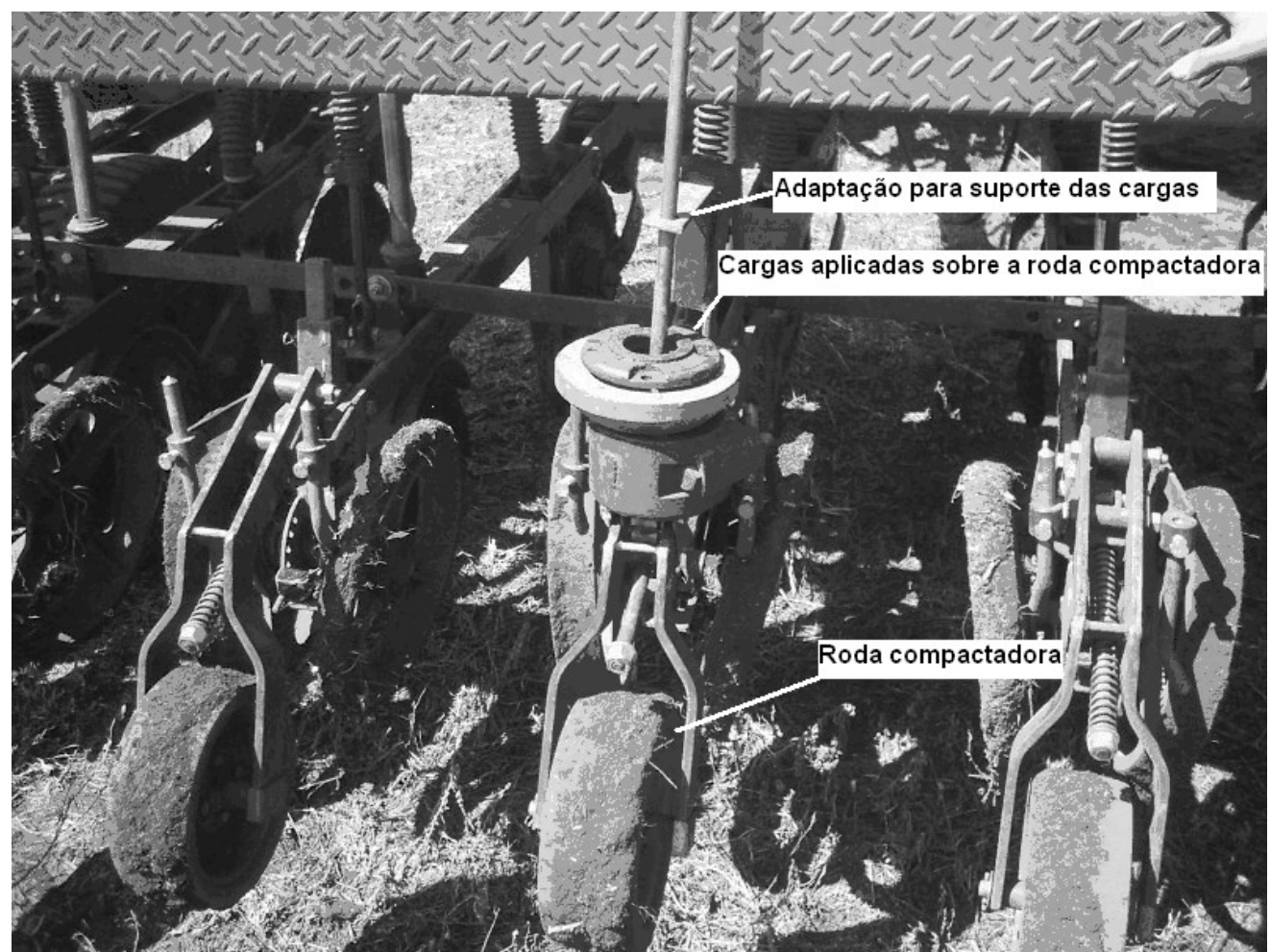

Figura 1 - Vista geral da adaptação realizada para mensurar as cargas aplicadas pela roda compactadora sobre a semente.

Tabela 1 - Síntese da análise de variância e do teste de médias para os valores médios do índice de velocidade de emergência (IVE), estandes inicial e final de plantas.

\begin{tabular}{|c|c|c|c|}
\hline Fator & & Estande Inicial & Estande Final \\
\hline Carga aplicada (C) & IVE & $\left(1.000\right.$ plantas $\left.\mathrm{ha}^{-1}\right)$ & $\left(1.000\right.$ plantas ha $\left.^{-1}\right)$ \\
\hline $\mathrm{C} 1$ & $14,83 \mathrm{a}$ & $232,6 \mathrm{a}$ & $221,5 \mathrm{a}$ \\
\hline $\mathrm{C} 2$ & $13,87 \mathrm{a}$ & $238,9 \mathrm{a}$ & $231,1 \mathrm{a}$ \\
\hline $\mathrm{C} 3$ & $12,38 \mathrm{a}$ & $216,3 \mathrm{a}$ & $194,9 \mathrm{a}$ \\
\hline $\mathrm{C} 4$ & $12,51 \mathrm{a}$ & $211,1 \mathrm{a}$ & $195,5 \mathrm{a}$ \\
\hline \multicolumn{4}{|l|}{ Profundidade $(\mathrm{P})$} \\
\hline $\mathrm{P} 1$ & $6,07 \mathrm{~b}$ & $202,8 \mathrm{~b}$ & $190,5 \mathrm{~b}$ \\
\hline $\mathrm{P} 2$ & $27,35 \mathrm{a}$ & $239,7 \mathrm{a}$ & $226,4 \mathrm{a}$ \\
\hline P3 & $6,79 \mathrm{~b}$ & $231,7 \mathrm{ab}$ & $215,4 \mathrm{ab}$ \\
\hline \multicolumn{4}{|l|}{ Teste F } \\
\hline $\mathrm{C}$ & $2,95^{\mathrm{NS}}$ & $1,71^{\mathrm{NS}}$ & $3,53^{\mathrm{NS}}$ \\
\hline $\mathrm{P}$ & $423,84^{* *}$ & $4,96^{*}$ & $4,90^{*}$ \\
\hline $\mathrm{C} \times \mathrm{P}$ & $2,04^{\mathrm{NS}}$ & $1,01^{\mathrm{NS}}$ & $1,21^{\mathrm{NS}}$ \\
\hline C.V. $(\%)$ & 17,52 & 15,52 & 15,80 \\
\hline
\end{tabular}

Em cada coluna, para cada fator, médias seguidas de mesmas letras minúsculas não diferem entre si, pelo teste de Tukey, a 5\% de probabilidade. ${ }^{\text {Ns: }}$ não-significativo $(\mathrm{P} \geq 0,005)$. *: significativo $(\mathrm{P}<0,05)$. **: significativo $(\mathrm{P}<0,01)$. C.V.: coeficiente de variação. 
Segundo Minami \& Haag (1989), a profundidade de semeadura é específica para cada espécie e quando adequada, propicia germinação e emergência de plântulas uniformes, as quais traduzem na obtenção de adequado estande. As excessivas profundidades de semeaduras podem impedir que a plântula ainda frágil emirja a superfície do solo. Por outro lado, se reduzidas, predispõem as sementes a qualquer variação ambiental, como excesso ou déficit hídrico ou térmico, as quais podem dar origem a plântulas mais fracas.

\section{Número de legumes por planta, número de grãos por legume, peso de 100 grãos e rendimento}

Na Tabela 2, observa-se que não houve diferenças significativas quanto ao número de legumes por planta, número de grãos por legume e peso de 100 grãos, tanto para as cargas aplicadas sobre a roda compactadora quanto para as profundidades de semeadura, e que não houve interação entre os fatores cargas aplicadas e profundidades de semeadura.

Silva et al. (2004), trabalhando com a cultura da soja observou que tanto as diferentes profundidades como as cargas aplicadas sobre a roda compactadora não influenciaram significativamente no número de legumes por planta.

Com relação ao rendimento médio por hectare (Tabela 2), verifica-se que não houve significância na interação, pois o rendimento da cultura do feijão não foi influenciado simultaneamente pelos fatores profundidade de semeadura e cargas aplicadas pela roda compactadora.

De acordo com essa Tabela, observa-se, também, que o fator profundidade de semeadura apresentou diferença significativa no rendimento da cultura, sendo que as profundidades $\mathrm{P} 2(5,0 \mathrm{~cm})$ e $\mathrm{P} 3(7,0 \mathrm{~cm})$ resultaram em maior rendimento quando comparadas com a profundidade $\mathrm{P} 1(3,0 \mathrm{~cm})$, fato que pode ser explicado pelo maior estande de plantas, obtidos nessas profundidades. Esse maior número de plantas, segundo Teixeira (2000) é fator condicionante da produtividade da cultura do feijoeiro, sendo que, a baixa densidade de semeadura está entre as principais causas dos baixos rendimentos nas lavouras de todo o Brasil.

Trabalhando com três profundidades de semeadura $(2,0 ; 4,0$ e $6,0 \mathrm{~cm})$ e quatro níveis de compactação do solo (0; 98; 196 e 294 N), na cultura do feijão, Sordi (2001) não encontrou diferenças de rendimento de grãos entre os tratamentos. Grotta et al. (2007), trabalhando com diferentes profundidades de semeadura e cargas aplicadas pela roda compactadora, também não encontraram diferenças significativas no rendimento da cultura da soja.

Grotta et al. (2008) avaliando o rendimento da cultura do amendoim em função de diferentes profundidades de semeadura e cargas aplicadas pela roda compactadora, verificaram que somente o fator profundidade de semeadura influenciou significativamente o rendimento da cultura, sendo que na maior profundidade $(8,0 \mathrm{~cm})$ houve redução

Tabela 2 - Síntese da análise de variância e do teste de médias para os valores médios dos componentes de rendimento.

\begin{tabular}{|c|c|c|c|c|}
\hline Fator & Número de & Número de & Peso de 100 & Rendimento \\
\hline Carga aplicada (C) & legumes por planta & grãos por legume & grãos (g) & médio $\left(\mathrm{kg} \mathrm{ha}^{-1}\right)$ \\
\hline $\mathrm{C} 1$ & $16,02 \mathrm{a}$ & 5,09 a & $26,26 \mathrm{a}$ & 2549,98 a \\
\hline $\mathrm{C} 2$ & $15,28 \mathrm{a}$ & $5,02 \mathrm{a}$ & $26,12 \mathrm{a}$ & $2687,01 \mathrm{a}$ \\
\hline $\mathrm{C} 3$ & $16,95 \mathrm{a}$ & $5,03 \mathrm{a}$ & $25,93 \mathrm{a}$ & $2377,75 \mathrm{a}$ \\
\hline $\mathrm{C} 4$ & $15,92 \mathrm{a}$ & $4,94 \mathrm{a}$ & $25,46 \mathrm{a}$ & $2577,75 \mathrm{a}$ \\
\hline \multicolumn{5}{|l|}{ Profundidade $(\mathrm{P})$} \\
\hline $\mathrm{P} 1$ & $16,96 \mathrm{a}$ & $5,18 \mathrm{a}$ & $26,07 \mathrm{a}$ & $2398,59 \mathrm{~b}$ \\
\hline $\mathrm{P} 2$ & $15,52 \mathrm{a}$ & $4,94 \mathrm{a}$ & $26,19 \mathrm{a}$ & 2843,03 a \\
\hline $\mathrm{P} 3$ & $15,64 \mathrm{a}$ & $4,94 \mathrm{a}$ & $25,56 \mathrm{a}$ & $2402,75 \mathrm{ab}$ \\
\hline \multicolumn{5}{|l|}{ Teste F } \\
\hline $\mathrm{C}$ & $0,68^{\mathrm{NS}}$ & $0,68^{\mathrm{NS}}$ & $0,92^{\mathrm{NS}}$ & $0,76^{\mathrm{NS}}$ \\
\hline $\mathrm{P}$ & $1,21^{\mathrm{NS}}$ & $1,21^{\mathrm{NS}}$ & $1,15^{\mathrm{NS}}$ & $4,03^{*}$ \\
\hline $\mathrm{C} \times \mathrm{P}$ & $1,55^{\mathrm{NS}}$ & $1,55^{\mathrm{NS}}$ & $0,94^{\mathrm{NS}}$ & $1,25^{\mathrm{NS}}$ \\
\hline C.V. $(\%)$ & 18,05 & 7,56 & 4,78 & 19,99 \\
\hline
\end{tabular}

Em cada coluna, para cada fator, médias seguidas de mesmas letras minúsculas não diferem entre si, pelo teste de Tukey, a 5\% de probabilidade. ${ }^{N S}$ : não-significativo $(\mathrm{P} \geq 0,005)$. *: significativo $(\mathrm{P}<0,05)$. **: significativo $(\mathrm{P}<0,01)$. C.V.: coeficiente de variação. 
significativa, quando comparada com as profundidades de 4,0 e $6,0 \mathrm{~cm}$. Os autores justificaram que tal fato pode ter ocorrido em razão do maior gasto energético realizado pelas plântulas de amendoim para romper essa camada mais espessa de solo, tornando a planta resultante mais frágil e debilitada, incorrendo em menor rendimento.

Para o fator compactação do solo sobre a semente (Tabela 2), constata-se que não houve diferença significativa entre todas as cargas aplicadas $(0,60,110$ e 190 N) indicando que a produtividade da cultura do feijão independe da carga aplicada pela roda compactadora, na ocasião do plantio. Esses resultados, entretanto, diferem dos obtidos por Coan et al. (1986) que, estudando a compactação, na cultura do feijão, em profundidade de semeadura constante de $7,5 \mathrm{~cm}$, observaram variação na produtividade da mesma.

\section{CONCLUSÕES}

Nas condições em que foi realizado esse trabalho, pode-se concluir que, a profundidade de semeadura influenciou significativamente o IVE, os estantes inicial e final de plantas e o rendimento médio da cultura do feijão.

A semeadura realizada nas profundidades de 5,0 e $7,0 \mathrm{~cm}$ obteve maior rendimento médio da cultura, quando comparada a profundidade de $3,0 \mathrm{~cm}$.

A interação profundidade $\mathrm{x}$ carga aplicada não apresentou correlação, mostrando que os fatores agiram independentemente.

As diferentes cargas aplicadas pela roda compactadora não influenciaram significativamente os parâmetros avaliados.

\section{AGRADECIMENTOS}

Os autores agradecem a Fundação Araucária pelo apoio financeiro para realização deste trabalho.

\section{REFERÊNCIAS BIBLIOGRÁFICAS}

BROWN, A.D.; DEXTER, A.R.; CHAMEN, W.C.T.; SPOOR, G. Effect of soil macroporosity and agregate size on seed-soil contact. Soil \& Tillage Research, Amsterdam, v.38, n.3, p.203-216, 1996.

COAN, O.; ORTOLANI, A.F.; PELLICER, C.A.P. Influência da profundidade de semeadura e compactação do solo sobre a semente, na emergência, desenvolvimento inicial e produtividade do feijoeiro (Phaseolus vulgaris L.). In: CONGRESSO BRASILEIRO DE ENGENHARIA AGRÍCOLA, 15., 1986, São Paulo. Anais... Botucatu: Sociedade Brasileira de Engenharia Agrícola, 1986. p.76-83.
CORTEZ, J.W.; CARVALHO FILHO, A.; SILVA, R.P.; FURLANI, C.E.A. Efeito de cargas verticais exercidas sobre rodas compactadoras na cultura do algodão (Gossypium hirsutum L.). FAZU em Revista, Uberaba, n.2, p.45-50, 2005.

DELLICER, C.A.P. Influência da profundidade de semeadura e compactação do solo sobre a semente na emergência, desenvolvimento inicial e produtividade do feijoeiro (Phaseolus vulgaris L.). 1984. 79f. Monografia (Graduação em Agronomia)-Faculdade de Ciências Agrárias e Veterinárias, Universidade Estadual Paulista, Jaboticabal, 1984.

\section{EMPRESA BRASILEIRA DE PESQUISA}

AGROPECUÁRIA. Centro Nacional de Pesquisa de Solos. Sistema brasileiro de classificação de solos. Rio de Janeiro, 1999.

FURLANI, C.E.A.; LOPES, A.; REZENDE, L.C.; SILVA, S.S.S. e; LEITE, M.A.S. Influência da compactação do solo na emergência das plântulas de milho a diferentes profundidades de semeadura. Engenharia na Agricultura, Viçosa, v.9, n.3, p.147-153, 2001.

GROTTA, D.C.; FURLANI, C.E.A.; SILVA, R.P.; REIS, G.N. dos; CORTEZ, J.W.; ALVES, P J. influência da profundidade de semeadura e da compactação do solo sobre a semente na produtividade do amendoim.

Ciência e Agrotecnologia, Lavras, v.32, n.2, p.547-552, 2008.

GROTTA, D.C.; FURLANI, C.E.A.; SILVA, R.P.; SANTOS, L. dos; CORTEZ, J.W.; REIS, G.N. dos. Cultura da soja em função da profundidade de semeadura e da carga vertical sobre a fileira de semeadura. Engenharia Agrícola, Jaboticabal, v.27, n.2, p.487-492, 2007.

MAGUIRE, J.D. Speed of germination-aid in selection and evaluation for seedling emergence and vigor. Crop science, Madison, v.2, n.1, p.176-177, 1962.

MINAMI, K.; HAAG, H.P. O tomateiro. Campinas: Fundação Cargil, 1989. 397p.

MODOLO, A.J.; FERNANDES, A.C.; SCHAEFER, C.E.G.; SILVEIRA, J.C.M. Efeito da compactação do solo sobre a emergência de plântulas de soja em sistema plantio direto. Ciência e Agrotecnologia, Lavras, v.32, n.4, p.1259-1265, 2008. 
NABI, G.; MULLINS, C.E.; MONTEMAYOR, M.B.; AKHTAR, M.S. Germination and emergence of irrigate cotton in Pakistan in relation to sowing depth and physical properties of the seedbed. Soil \& Tillage Research, Amsterdam, v.59, n.2, p.33-44, 2000.

PHILLIPS, R.E.; KIRKHAN, D. Mechanical impedance and corn seedling root growth. Soil Science Society America Proceedings, Madison, v.26, n.4, p.319-322, 1962.

REZENDE, R.; FREITAS, P.S.L.; MANTOVANI, E.C.; FRIZZONE, J.A. Função de produção da cultura do milho e do feijão para diferentes lâminas e uniformidade de aplicação de água. Acta Scientiarum Agronomy, Maringá, v.26, n.4, p.503-511, 2004.

SILVA, F.M.; ORTOLANI, F.M.; DANIEL, L.A. Rodas compactadoras de semeadoras-adubadoras: influência no condicionamento físico do solo na região de semeadura. In: CONGRESSO BRASILEIRO DE ENGENHARIA AGRÍCOLA, 20., 1991, Londrina. Anais... Londrina: SBEA, 1991. p.1.126-1.146.

SILVA, R.P. Efeito de rodas compactadoras submetidas a cargas verticais em profundidades de semeadura nas características agronômicas do milho (Zea mays $L$.).
2002. 101f. Tese (Doutorado em Agronomia)-

Universidade Estadual Paulista, Jaboticabal, 2002.

SILVA, R.P.; CORÁ, J.E.; FURLANI, C.E.A.; LOPES, A. Efeito da profundidade de semeadura e de rodas compactadoras submetidas a cargas verticais na temperatura e no teor de água do solo durante a germinação de sementes de milho. Ciência e Agrotecnologia, Lavras, v.32, n.3, p.929-937, 2008.

SILVA, R.P.; TEIXEIRA, F.A.C.; CAMPOS, M.A.O. Efeito da profundidade de semeadura e da carga sobre a roda compactadora no desenvolvimento da soja (Glycine max). Engenharia na Agricultura, Viçosa, v.12, n.3, p.169-176, 2004.

SORDI, F. Efeito da profundidade de semeadura e da compactação do solo sobre a semente na cultura do feijoeiro (Phaseolus vulgaris). 2001. 50f. Monografia (Graduação em Agronomia)-Faculdade de Ciências Agrárias e Veterinárias, Universidade Estadual Paulista, Jaboticabal, 2001.

TEIXEIRA, I.R.; ANDRADE, M.J.B. de; CARVALHO, J.G. de; MORAIS, A.R. de; CORRÊA, J.B.D. Resposta do feijoeiro (Phaseolus vulgaris L. cv. Pérola) a diferentes densidades de semeadura e doses de nitrogênio. Ciência e Agrotecnologia, Lavras, v.24, n.2, p.399-408, 2000. 